\title{
Liver surgery prolongs the survival of patients with gastrointestinal stromal tumor liver metastasis: a retrospective study from a single
} center

This article was published in the following Dove Press journal:

Cancer Management and Research

\author{
Binyi Xiao* \\ Jianhong Peng* \\ Jinghua Tang* \\ Rongxin Zhang \\ Cong $\mathrm{Li}$ \\ Junzhong Lin \\ Peirong Ding \\ Desen Wan \\ Zhizhong Pan \\ Xiaojun Wu
}

Sun Yat-sen University Cancer Center, State Key Laboratory of Oncology in South China, Collaborative Innovation Center of Cancer Medicine,

Guangzhou 510060, China

*These authors contributed equally to this work
Correspondence: Xiaojun Wu; Zhizhong Pan

Sun Yat-sen University Cancer Center, State Key Laboratory of Oncology in South China, Collaborative Innovation Center of Cancer Medicine, 65I

Dongfeng Road East, Guangzhou 510060 , PR China

Tel +862087343124

Fax +862087343637

Email wuxj@sysucc.org.cn; panzhzh@ sysucc.org.cn
Objectives: Gastrointestinal stromal tumor (GIST) liver metastasis (GLM) is a special subset of advanced GIST, because its lesions are easier to define and assess. We aim to determine the role of liver metastasectomy for patients with GLM in the era of tyrosine kinase inhibitor (TKI) therapy. Methods: We reviewed patients with metastatic GIST who received surgery or other treatments in Sun Yat-sen University Cancer Center between January 1991 and December 2017. Patients with metastases confined to the liver and with no previous metastasis to other locations were included into the study and were classified into surgical and non-surgical groups. All patients received $400 \mathrm{mg} / \mathrm{d}$ imatinib after the operation. We compared progression-free survival (PFS) and overall survival (OS) between the two groups.

Results: A total of 102 patients were included into the study. Of them, 21 (20.1\%) underwent surgery for liver metastases and $81(79.9 \%)$ received TKI therapy alone. During the operation, six patients received radiofrequency ablation for suspicious or unresectable lesions. Three-year PFS rate was $77.5 \%$ in the surgical group and $65.5 \%$ in the non-surgical group ( $P=0.027)$; 5 -year OS rate was $85.7 \%$ and $59.6 \%$, respectively $(P=0.008)$. About $22.1 \%$ of patients had metastases of less than three in the surgical group, while the rate was $42.9 \%$ in the non-surgical group $(P=0.011)$. Patients with metastases of less than three had longer PFS than those with three or more, with a 3 -year PFS rate of $72.8 \%$ and $65.8 \%$, respectively $(P=0.019)$. But their difference in 5-year OS rate was not significant $(91.7 \%$ vs $55.3 \%, P=0.08)$.

Conclusion: Followed by continuous TKI therapy, R0 surgery significantly prolongs the survival of patients with GLM, regardless of the extent of disease or the phase of metastasis.

Keywords: gastrointestinal stromal tumor, liver metastasis, surgery, metastasectomy

\section{Introduction}

Gastrointestinal stromal tumor (GIST) is the most common sarcoma of the digestive tract, with an estimated incidence of 11.0-21.0 per million worldwide. ${ }^{1,2}$ GIST ranges in malignancy from small nodules that pursuit a benign course to bulky sarcoma with metastatic potential. Metastasis occurs in 15\%-50\% of patients at the time of diagnosis, making complete resection difficult. ${ }^{3,4}$ For patients with localized high-risk GIST, despite complete resection, nearly $40 \%$ suffer tumor relapse within 2 years. The liver and peritoneum are the most common sites of metastasis, accounting for about $65 \%$ and $20 \%$ of the total, respectively. ${ }^{3}$

Imatinib, a tyrosine kinase inhibitor (TKI), is the first-line treatment for advanced GIST. In clinical trials, it achieved high disease control rates and significantly prolonged 
survival. ${ }^{5,6}$ However, despite its potent efficacy, resistance occurs in nearly $50 \%$ of patients after a treatment of 2 years. The limitation of TKI therapy has prompted the reassessment of surgery for advanced GIST, but opinions differ. Some suggested that only patients with stable or limited progression disease on TKI therapy could benefit from debulking surgery, ${ }^{7}$ whereas others found that when complete resection could be achieved, even those with progressing disease would benefit. ${ }^{8}$ In a Korean study, however, tumor burden instead of surgery was found to be a predictor of survival. ${ }^{9}$

However, those studies dealt with patients with metastases in different locations. But from an anatomical perspective, metastases in the liver are easier to define and assess than those in the peritoneum. This feature gives GIST liver metastases (GLM) greater possibility of complete resection, distinguishing it from other metastatic GIST. Indeed, in a European study, patients with GLM had higher rate of R0 resection and showed the longest survival. ${ }^{10}$ Therefore, GLM may represent a special subset of advanced GIST where surgery has a greater role to play.

Although there are some studies dealing with patients with GLM, their baseline values and treatment strategies varied greatly: some patients had previous metastasis before spreading to the liver, and some did not receive TKI therapy after the operation. ${ }^{11,12}$ These variations were associated with a poor survival. ${ }^{13}$ Therefore, it is necessary to reassess the value of surgery in this subset of patients with a more balanced dataset. The aim of this study was to determine whether metastasectomy improves the survival of patients with GLM in the era of TKI therapy.

\section{Patients and methods}

\section{Patient selection}

We reviewed patients with metastatic GIST who received surgery or other treatments in Sun Yat-sen University Cancer Center between January 1991 and December 2017. Pathological diagnoses were performed or confirmed in our center. This retrospective study was approved by the institutional review board of Sun Yat-sen University Cancer Center, and written informed consent was obtained from all patients.

A total of 102 patients with liver-only metastases were included in this study. They were defined as those with metastases restricted to the liver, regardless of whether or not experiencing further dissemination to other locations. Patients with previous metastasis to the peritoneum, lung, bone, or other organs were excluded. Of the 102 patients, 21 underwent surgery for liver metastases (surgical group), while the other 81 received TKI therapy alone (non-surgical group). According to the time of occurrence, liver metastasis was classified into synchronous and metachronous. Synchronous liver metastasis was defined as metastasis that occurred at diagnosis or within 3 months after the resection of primary tumor, while metachronous liver metastasis was defined as one that occurred beyond 3 months after the resection (RDDA2018000853).

\section{Tumor characteristics}

Risk stratification for primary tumor was performed using NIH criteria in $51(50 \%)$ patients who had no metastases at diagnosis. ${ }^{22}$ The first radiological reports that described liver metastases were retracted in 85 patients but were unavailable in the other 17. According to these reports, the extent of liver metastases was classified as limited, if there were less than three detectable diseases, or as generalized, if there were three or more. Diameters of the largest metastases were depicted in 79 patients, with a median of $4.4 \mathrm{~cm}$ (range $1.0-15.0 \mathrm{~cm}$ ). Fifty-four patients were tested for mutational status of genes $C$-KIT and PDGFRA by using primary tumor.

\section{Surgical management}

Liver metastasectomy was performed in our center or other hospitals. For patients with synchronous metastases, resection of primary tumor was performed concurrently. Surgical procedures for liver lesions included partial resection, subsegmentectomy, and segmentectomy. Radiofrequency ablation was applied for suspicious or unreachable lesions to achieve NED (no evidence of disease). For patients receiving preoperative imatinib, all surgeries were performed when the diseases were responsive or stable.

\section{Systemic treatment}

All patients received $400 \mathrm{mg} / \mathrm{d}$ imatinib as first-line treatment after the operation. When disease progressed, they were prescribed second-line regimen, either with high-dose imatinib (600 mg/d or $800 \mathrm{mg} / \mathrm{d}$ ) or sunitinib (37.5 mg/d). CT scan was scheduled every 6 months. Evaluation of response was performed according to RECIST criteria. $^{23}$

\section{End points and statistics}

Progression-free survival (PFS) was defined as the time between liver metastasis and disease progression or death from any cause, whichever came first. Overall survival (OS) was calculated from the time of liver metastasis to the time of death from any cause. The comparison of clinicopathological parameters between surgical and non-surgical groups was performed using chi-squared or Mann-Whitney $U$ test, as 
appropriate. Prognostic factors were analyzed by univariate logistic regression. Parameters with $P<0.1$ were included into multivariate analysis. All survival curves were calculated using Kaplan-Meier methods. Two-sided $P$-value less than 0.05 was considered significant.

\section{Results}

\section{Demographic data}

Demographic data are listed in Table 1. A total of 102 patients were included into the study, with $71(69.6 \%)$ males and 31 (30.4\%) females. Twenty-one (20.1\%) patients underwent surgery for liver metastases, while 81 (79.9\%) patients were

Table I Patient characteristics

\begin{tabular}{|c|c|c|}
\hline Parameters & Cases $(n=102)$ & Percent \\
\hline \multicolumn{3}{|l|}{ Sex } \\
\hline Male & 71 & 69.6 \\
\hline Female & 31 & 30.4 \\
\hline \multicolumn{3}{|l|}{ Age (years) } \\
\hline Median & 52 & - \\
\hline Range & $17-83$ & - \\
\hline$<60$ & 74 & 72.5 \\
\hline$\geq 60$ & 28 & 27.5 \\
\hline \multicolumn{3}{|l|}{ Primary sites } \\
\hline Stomach & 38 & 37.3 \\
\hline Duodenum & 12 & 11.8 \\
\hline Intestine & 38 & 37.3 \\
\hline Colon and rectum & 2 & 2.0 \\
\hline Others & 12 & 11.8 \\
\hline \multicolumn{3}{|l|}{ Risk stratification ${ }^{\mathrm{a}}$} \\
\hline Middle & 4 & 3.9 \\
\hline High & 47 & 46.1 \\
\hline Metastatic & 51 & 48.0 \\
\hline \multicolumn{3}{|l|}{ Metastatic phase } \\
\hline Synchronous & 51 & 50.0 \\
\hline Metachronous & 51 & 50.0 \\
\hline \multicolumn{3}{|l|}{ No. of metastases ${ }^{b}$} \\
\hline$<3$ & 24 & 28.2 \\
\hline$\geq 3$ & 61 & 71.8 \\
\hline \multicolumn{3}{|l|}{ Diameter $(\mathrm{cm})^{\mathrm{c}}$} \\
\hline Mean & 4.4 & - \\
\hline Range & $1.0-15.0$ & - \\
\hline \multicolumn{3}{|l|}{ Genotype $^{d}$} \\
\hline C-KIT II exon & 42 & 77.8 \\
\hline C-KIT 9 exon & 5 & 9.3 \\
\hline Wild-type & 6 & $\mathrm{II} . \mathrm{I}$ \\
\hline PDGFRA & $\mathrm{I}$ & 1.9 \\
\hline \multicolumn{3}{|l|}{ Treatment } \\
\hline Surgery & 21 & 20.1 \\
\hline Non-surgery & 81 & 79.9 \\
\hline
\end{tabular}

Notes: aPatients were stratified at diagnosis and according to $\mathrm{NIH}$ cretiria; ${ }^{b}$ radiological assessment for liver metastases was not available in 17 patients; 'diameters of the largest metastases, data not available in 23 patients; dgenotype tests for primary tumor were not performed in 48 patients.

Abbreviation: $\mathrm{NIH}$, National Institutes of Health. treated with TKI drugs. Fifty-one (51\%) patients had synchronous metastases, and $51(50 \%)$ had metachronous metastases. Twenty-four (28.2\%) patients had metastases of less than three, while $61(71.8 \%)$ had three or more metastases.

\section{Comparison between surgical and non- surgical groups}

Table 2 shows clinicopathological features of surgical and non-surgical groups. The number of liver metastases differs significantly between the two groups. About $42.9 \%$ of patients in surgical group had metastases of less than three, while the rate was $22.1 \%$ in non-surgical group $(P=0.011)$. A marked difference was noted in the size of metastases, with $10(62.5 \%)$ and $31(49.2 \%)$ patients having a largest

Table 2 Comparison of clinicopathological parameters between surgical and non-surgical groups

\begin{tabular}{|c|c|c|c|}
\hline Parameters & \begin{tabular}{|l} 
Surgical \\
group
\end{tabular} & \begin{tabular}{|l} 
Non-surgical \\
group
\end{tabular} & $P$-value \\
\hline \multicolumn{4}{|l|}{ Sex } \\
\hline Male & II (52.4) & 60 (74.I) & 0.054 \\
\hline Female & $10(47.6)$ & $21(25.9)$ & \\
\hline \multicolumn{4}{|l|}{ Age (years) } \\
\hline$<60$ & 15 (7I.4) & 59 (72.8) & 0.897 \\
\hline$\geq 60$ & $6(28.6)$ & $22(27.2)$ & \\
\hline \multicolumn{4}{|l|}{ No of metastases ${ }^{a}$} \\
\hline$<3$ & $9(42.9)$ & $15(22.1)$ & 0.011 \\
\hline$\geq 3$ & $8(38.1)$ & $53(77.9)$ & \\
\hline \multicolumn{4}{|l|}{ Diameter $(\mathrm{cm})^{\mathrm{b}}$} \\
\hline$<4.5$ & $10(62.5)$ & $31(49.2)$ & 0.342 \\
\hline$\geq 4.5$ & $6(37.5)$ & $32(50.8)$ & \\
\hline \multicolumn{4}{|l|}{ Primary sites } \\
\hline Stomach & $8(38.1)$ & $30(37.0)$ & 0.141 \\
\hline Intestine & II (52.4) & $27(33.3)$ & \\
\hline Others & $2(9.5)$ & $24(29.7)$ & \\
\hline \multicolumn{4}{|l|}{ Genotype $^{c}$} \\
\hline C-KIT II & $12(57.1)$ & $30(37.0)$ & 0.808 \\
\hline Others & $3(14.3)$ & $9(\mathrm{II} . \mathrm{I})$ & \\
\hline \multicolumn{4}{|l|}{ Metastatic phase } \\
\hline Synchronous & II (52.4) & $40(49.4)$ & 0.807 \\
\hline Metachronous & $10(47.6)$ & $4 I(50.6)$ & \\
\hline \multicolumn{4}{|c|}{ Response to first-line Imatinib } \\
\hline CR & $\mathrm{I}(4.8)$ & $3(3.7)$ & 0.285 \\
\hline PR & 7 (33.3) & $4 I(50.6)$ & \\
\hline SD & $13(61.9)$ & $35(43.2)$ & \\
\hline PD & $0(0)$ & $2(2.5)$ & \\
\hline \multicolumn{4}{|l|}{ Second-line $\mathrm{TKI}^{\mathrm{d}}$} \\
\hline Yes & $16(76.2)$ & $47(58.0)$ & 0.127 \\
\hline No & $5(23.8)$ & $34(42.0)$ & \\
\hline
\end{tabular}

Notes: ${ }^{a}$ Radiological reports of 17 patients are not available; bdiameter of the largest metastases, data not available in 23 patients; 'genotype tests for primary

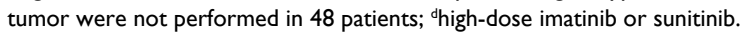

Abbreviations: CR, complete response; PD, progressive disease; PR, partial response; SD, standard disease; TKI, tyrosine kinase inhibitor. 
metastases of less than $4.5 \mathrm{~cm}$ in diameter in the two groups, respectively, but the difference was not significant $(P=0.342)$. There was no significant difference in sex, age, primary sites, metastatic phase, and response to imatinib.

For patients undergoing metastasectomy, 14 (66.5\%) received preoperative imatinib, and all of them achieved partial response or standard disease. Surgical procedures included partial resection in eleven patients, partial resection+ ablation in four, subsegmentectomy in three, segmentectomy in three, and segmentectomy+ ablation in two. Fifteen patients had complete resection ( $\mathrm{R} 0$ resection), and six patients achieved NED with the help of intraoperative radiofrequency ablation. Six patients experienced disease progression because of discontinuation of imatinib. All of them regained control after resuming imatinib, but five of them later developed resistance and were treated with second-line regimens (Table 3).

\section{Survival analysis}

Survival analysis is shown in Figure 1. The 3-year PFS rate was $77.5 \%$ in the surgical group and $65.5 \%$ in the non-surgical group ( $P=0.027)$, and the 5 -year OS rate was $85.7 \%$ and $59.6 \%$ for the two groups, respectively $(P=0.008)$. Notably, patients with metastases of less than three had longer PFS than those with three or more, with a 3 -year PFS rate of $72.8 \%$ and $65.8 \%$, respectively $(P=0.019)$. But their difference in 5 -year OS rate was not significant ( $91.7 \%$ vs $55.3 \%, P=0.08$ ). One patient in the surgical group died from uremia that was

Table 3 Patients undergoing metastectomy $(n=2 I)$

\begin{tabular}{|l|l|l|l|}
\hline Parameters & & n & $\%$ \\
\hline Preoperative IM & & & \\
\hline & No & 7 & 33.4 \\
\hline & Yes & I4 & 66.7 \\
\hline Surgical procedures & & & \\
\hline & Partial resection & II & 52.4 \\
\hline & Partial resection + ablation & 4 & 19.0 \\
\hline & Subsegmentectomy & 3 & 14.3 \\
\hline & Segmentectomy & 3 & 14.3 \\
\hline & Segmentectomy + ablation & 2 & 9.5 \\
\hline Surgical outcome & & & \\
\hline & & & \\
\hline & R0 & I5 & 71.4 \\
\hline Survival outcome & NED & 6 & 28.6 \\
\hline & & & \\
\hline & With tumor & 6 & 60.0 \\
\hline Gene mutation & Death & I & 40.0 \\
\hline & & & \\
\hline & C-KIT II & II & 52.4 \\
\hline & Unknown & I0 & 47.6 \\
\hline
\end{tabular}

Notes: ${ }^{\text {aPatients }}$ who received ablation concurrently; ${ }^{\mathrm{b}}$ patients who were not tested for genotypes.

Abbreviations: NED, no evidence of disease; IM, imatinib. considered unrelated to the treatment. In multivariate analysis, although there was a tendency that metastasectomy might be an independent factor of PFS and OS, the results were not significant ( $P=0.064$ and 0.095 , respectively). Neither surgery nor the number of metastases was an independent factor of survival (Table 4).

\section{Discussion}

In this study, we found that when combined with TKI therapy, metastasectomy significantly prolonged the survival of patients with GLM. Our study focused on a special subset of GIST patients from a single center and offered an insight into the role of surgery in this setting.

The advent of imatinib has made debulking surgery for advanced GIST controversial. However, there is evidence suggesting benefits of such surgery. One possible reason is that surgery can remove tumors that contain secondary mutations, making imatinib effective again for these patients. As suggested in previous studies, tumors progressing on TKI therapy were more likely to contain secondary mutations than those responsive to the treatment. ${ }^{14,15}$

GLM represents a special subgroup of advanced GIST. Hepatic lesions are easier to identify than metastases to other organs, and therefore are more amenable to surgery. In 2016 , researchers found that when complete resection is achieved, patients with GLM may benefit from surgery, with survival rate at 5 years exceeding $80 \%$. But in that study, $10 \%$ of the patients had extra-hepatic metastases before surgery, and $25 \%$ did not receive TKI therapy after surgery, which was crucial for prolonging patient survival. ${ }^{11,13}$ In our study, all patients received TKI therapy after surgery. We confirmed that complete resection of liver metastases was associated with better PFS and OS. The 5-year OS rate in surgical group was $85.7 \%$. This rate was comparable to that in localized GIST, where the rate was $85.3 \%$ for patients receiving imatinib for 1 year after complete resection. ${ }^{16}$ The promising result indicates that R0 surgery, when followed by continuous TKI therapy, may be curative for patients with GLM.

In six patients for whom complete resection was not feasible, we used radiofrequency ablation to destroy residual or suspicious lesions. All patients achieved NED, and none of them showed recurrence during follow-up. It follows that when NED is achievable, ablation can produce the same survival benefits as surgery does. The result was echoed by a previous study, in which patients with GLM had a 5-year OS rate of $87.7 \%$ after liver surgery combined with intraoperative ablation. Radiofrequency ablation was effective and safe for this group of patients. ${ }^{17,18}$ 
A

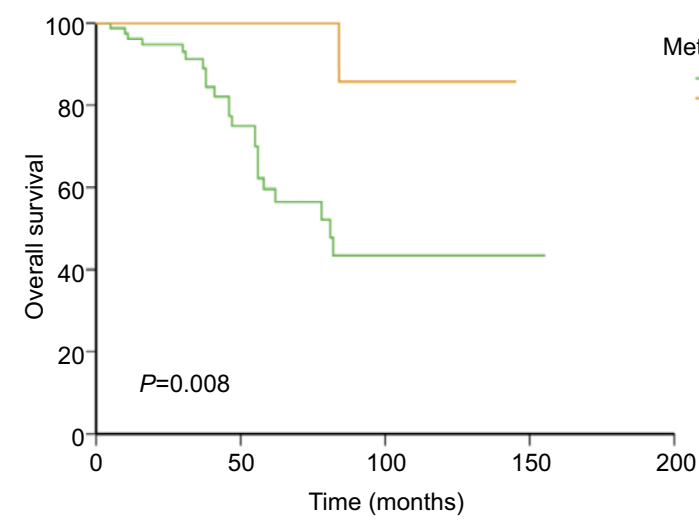

C

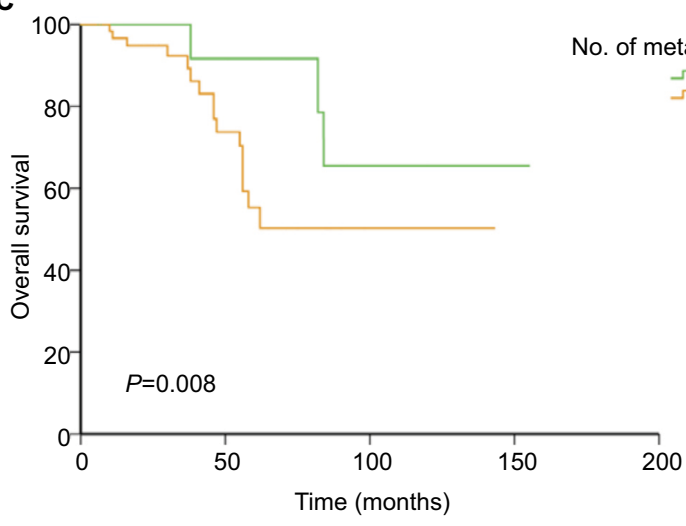

B
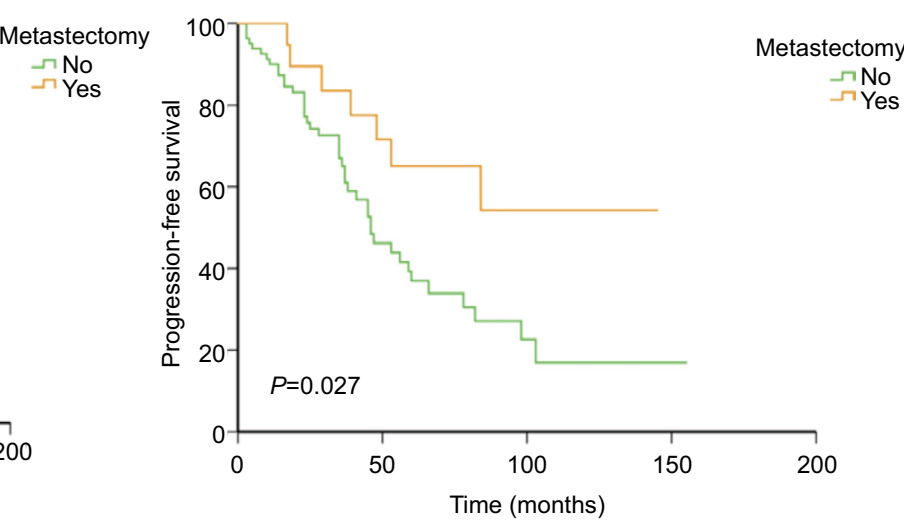

D

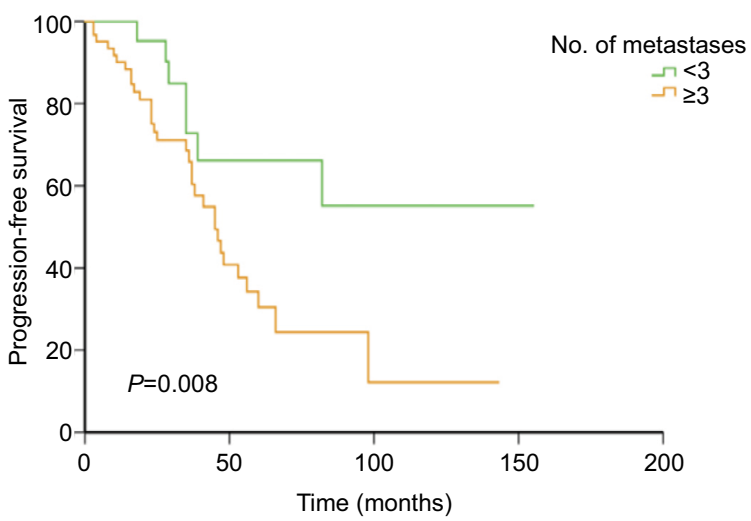

Figure I Comparison of survival between different groups.

Notes: (A) Overall survival curves for patients in surgical and non-surgical groups; (B) PFS curves for patients in surgical and non-surgical groups; (C) overall survival curves for patients with liver metastases $<3$ and those with $\geq 3$; (D) PFS curves for patients with liver metastases $<3$ and those with $\geq 3$.

Abbreviation: PFS, progression-free survival.

Table 4 Correlation of clinicopathological factors with PFS and OS

\begin{tabular}{|c|c|c|c|c|c|c|c|c|}
\hline \multirow[t]{2}{*}{ Parameters } & \multicolumn{4}{|l|}{ PFS } & \multicolumn{4}{|l|}{ OS } \\
\hline & Median & 3 years & $\begin{array}{l}P \text {-value } \\
\text { (univariate) }\end{array}$ & $\begin{array}{l}P \text {-value } \\
\text { (multivariate) }\end{array}$ & Median & 5 years & $\begin{array}{l}P \text {-value } \\
\text { (univariate) }\end{array}$ & $\begin{array}{l}\text { P-value } \\
\text { (multivariate) }\end{array}$ \\
\hline \multicolumn{9}{|l|}{ Sex } \\
\hline Male & 82.0 & 85.7 & 0.059 & 0.144 & 84.5 & 62.2 & 0.143 & - \\
\hline Female & NYR & 89.4 & & & NYR & 93.3 & & \\
\hline \multicolumn{9}{|l|}{ Age (years) } \\
\hline$<60$ & NYR & 84.8 & 0.863 & - & NYR & 71.8 & \begin{tabular}{|l|}
0.462 \\
\end{tabular} & - \\
\hline$\geq 60$ & 82.0 & 92.0 & & & 82.0 & 59.2 & & \\
\hline \multicolumn{9}{|l|}{ Primary sites } \\
\hline Stomach & NYR & 88.3 & 0.956 & - & NYR & 69.3 & 0.958 & - \\
\hline Intestine & 82.0 & 88.7 & & & 84.5 & 72.7 & & \\
\hline Others & NYR & 80.8 & & & NYR & 82.3 & & \\
\hline \multicolumn{9}{|l|}{ Metastatic phase } \\
\hline Synchronous & 46 & 65.4 & 0.140 & - & NYR & 57.1 & 0.239 & - \\
\hline Metachronous & 59 & 73.2 & & & NYR & 77.0 & & \\
\hline \multicolumn{9}{|l|}{ Metastectomy } \\
\hline Yes & NYR & 77.5 & 0.027 & 0.064 & NYR & 85.7 & 0.008 & 0.095 \\
\hline No & NYR & 65.0 & & & 81.0 & 59.6 & & \\
\hline \multicolumn{9}{|l|}{ No of metastases } \\
\hline$<3$ & NYR & 72.8 & 0.019 & 0.142 & NYR & 91.7 & 0.080 & 0.185 \\
\hline$\geq 3$ & 45.0 & 65.8 & & & NYR & 55.3 & & \\
\hline
\end{tabular}

Abbreviations: PFS, progression-free survival; OS, overall survival; NYR, not yet reached. 
Both surgery and ablation, or their combination, serve the purpose of reducing tumor burden to the greatest extent, leaving no visible tumor cell to develop resistance during subsequent TKI therapy. However, unlike patients with localized GIST, for whom optimal duration of adjuvant TKI therapy remains debatable, patients with GLM seem to have to maintain the treatment lifelong. In a stage III clinical study, Kanda et al compared the efficacy of surgery with imatinib for GIST patients with resectable hepatic lesions and found that all patients experienced tumor recurrence after R0 resection, with a median RFS of only 145 days and a 3-year RFS rate of $16.7 \%$. Hence, it was concluded that surgery alone, even achieving R0, was not curative for patients with GLM. Imatinib remained the main treatment for this group of patients. ${ }^{19}$ In our study, six patients who had discontinued imatinib after the operation developed new lesions rapidly, while the others stayed tumor-free at the last follow-up. This contrast suggests that continuous TKI therapy may be more important than surgery for a long-term survival.

Several other factors may affect patient survival, including patient age, baseline performance status, and pretreatment tumor burden., ${ }^{3,920}$ In our study, patients with less tumors were associated with longer PFS and OS, which suggested that the survival advantage observed in the surgical group may be attributed to its lower tumor burden. However, some argued that tumor burden affects survival through its impact on resectability, and as long as complete resection can be achieved, the impact can be overridden by surgery. ${ }^{10}$

Interestingly, unlike colorectal cancer liver metastasis, where synchronous metastasis is considered to be more aggressive, metastatic phase seems not to affect patient outcome in GIST. In our study, PFS and OS did not differ between the two phases. Similar result was observed in a previous study. ${ }^{12}$ One possible reason is that gene mutation weighs more heavily in the development and progression of GIST than in that of colorectal cancer, with secondary mutation found in as high as $80 \%$ of progressed GIST lesions. ${ }^{14}$ Another reason is that TKI therapy for GIST is far more effective than chemotherapy for colorectal cancer, and the impact of metastatic phase is overshadowed.

Our study has some limitations. First, as a retrospective study, selection bias is unavoidable. Patients with less metastases were more likely to be selected for surgery. Second, due to the scarcity of this population, our study had a small sample. Third, the credibility of the study is subject to patients' compliance with treatment protocol. Some patients did not receive regular radiological examination, which might postpone the documentation of progression, and some patients discontinued treatment without consultation, which might impair their long-term survival. ${ }^{21}$

\section{Conclusion}

When $\mathrm{R} 0$ resection can be achieved, surgery is recommended for patients with GLM, regardless of the extent of disease or the phase of metastasis. Future studies with larger dataset and more balanced information are warranted.

\section{Acknowledgments}

Special thanks are due to Sherry Young, without whose support this paper would never have been completed. The study was funded by National Natural Science Foundation of China (No 81502459 and No 81772595) and Science and Technology Project in Guangdong Province (No 2013B021800146).

\section{Disclosure}

The authors report no conflicts of interest in this work.

\section{References}

1. Tryggvason G, Gíslason HG, Magnússon MK, Jónasson JG. Gastrointestinal stromal tumors in Iceland, 1990-2003: the icelandic GIST study, a population-based incidence and pathologic risk stratification study. Int J Cancer. 2005;117(2):289-293.

2. Chan KH, Chan CW, Chow WH, et al. Gastrointestinal stromal tumors in a cohort of Chinese patients in Hong Kong. World J Gastroenterol. 2006;12(14):2223-2228.

3. Dematteo RP, Lewis JJ, Leung D, Mudan SS, Woodruff JM, Brennan MF. Two hundred gastrointestinal stromal tumors: recurrence patterns and prognostic factors for survival. Ann Surg. 2000;231(1):51-58.

4. Nilsson B, Bümming P, Meis-Kindblom JM, et al. Gastrointestinal stromal tumors: the incidence, prevalence, clinical course, and prognostication in the preimatinib mesylate era - a population-based study in western Sweden. Cancer. 2005;103(4):821-829.

5. Demetri GD, von Mehren M, Blanke CD, et al. Efficacy and safety of imatinib mesylate in advanced gastrointestinal stromal tumors. $N$ Engl J Med. 2002;347(7):472-480.

6. Verweij J, Casali PG, Zalcberg J, et al. Progression-free survival in gastrointestinal stromal tumours with high-dose imatinib: randomised trial. Lancet. 2004;364(9440):1127-1134.

7. Raut CP, Posner M, Desai J, et al. Surgical management of advanced gastrointestinal stromal tumors after treatment with targeted systemic therapy using kinase inhibitors. J Clin Oncol. 2006;24(15):2325-2331.

8. Hasegawa J, Kanda T, Hirota S, et al. Surgical interventions for focal progression of advanced gastrointestinal stromal tumors during imatinib therapy. Int J Clin Oncol. 2007;12(3):212-217.

9. An HJ, Ryu MH, Ryoo BY, et al. The effects of surgical cytoreduction prior to imatinib therapy on the prognosis of patients with advanced GIST. Ann Surg Oncol. 2013;20(13):4212-4218.

10. Bauer S, Rutkowski P, Hohenberger P, et al. Long-term follow-up of patients with GIST undergoing metastasectomy in the era of imatinib - analysis of prognostic factors (EORTC-STBSG collaborative study). Eur J Surg Oncol. 2014;40(4):412-419.

11. Seesing MF, Tielen R, van Hillegersberg R, et al. Resection of liver metastases in patients with gastrointestinal stromal tumors in the imatinib era: A nationwide retrospective study. Eur J Surg Oncol. 2016;42(9):1407-1413.

12. Shi YN, Li Y, Wang LP, et al. Gastrointestinal stromal tumor (GIST) with liver metastases: An 18-year experience from the GIST cooperation group in North China. Medicine. 2017;96(46):e8240. 
13. Sato S, Tsujinaka T, Yamamoto K, et al. Primary surgery as a frontline treatment for synchronous metastatic gastrointestinal stromal tumors: an analysis of the Kinki GIST registry. Surg Today. 2016;46(9):1068-1075.

14. Liegl B, Kepten I, Le C, et al. Heterogeneity of kinase inhibitor resistance mechanisms in GIST. J Pathol. 2008;216(1):64-74.

15. Yeh CN, Chen TW, Tseng JH, et al. Surgical management in metastatic gastrointestinal stromal tumor (GIST) patients after imatinib mesylate treatment. J Surg Oncol. 2010;102(6):599-603.

16. Dematteo RP, Ballman KV, Antonescu CR, et al. Adjuvant imatinib mesylate after resection of localised, primary gastrointestinal stromal tumour: a randomised, double-blind, placebo-controlled trial. Lancet. 2009;373(9669):1097-1104.

17. Jung JH, Won HJ, Shin YM, Kim PN. Safety and Efficacy of Radiofrequency Ablation for Hepatic Metastases from Gastrointestinal Stromal Tumor. J Vasc Interv Radiol. 2015;26(12):1797-1802.

18. Yoon IS, Shin JH, Han K, et al. Ultrasound-Guided Intraoperative Radiofrequency Ablation and Surgical Resection for Liver Metastasis from Malignant Gastrointestinal Stromal Tumors. Korean J Radiol. 2018;19(1):54-62.
19. Kanda T, Masuzawa T, Hirai T, et al. Surgery and imatinib therapy for liver oligometastasis of GIST: a study of Japanese Study Group on GIST. Jpn J Clin Oncol. 2017;47(4):369-372.

20. Blanke CD, Demetri GD, von Mehren M, et al. Long-term results from a randomized phase II trial of standard- versus higher-dose imatinib mesylate for patients with unresectable or metastatic gastrointestinal stromal tumors expressing KIT. J Clin Oncol. 2008;26(4): 620-625.

21. Blay JY, Pérol D, Le Cesne A. Imatinib rechallenge in patients with advanced gastrointestinal stromal tumors. Ann Oncol. 2012;23(7): 1659-1665.

22. Joensuu H. Risk stratification of patients diagnosed with gastrointestinal stromal tumor. Hum Pathol. 2008;39(10):1411-1419.

23. Therasse P, Arbuck SG, Eisenhauer EA et al. New guidelines to evaluate the response to treatment in solid tumors. European Organization for Research and Treatment of Cancer, National Cancer Institute of the United States, National Cancer Institute of Canada. J Natl Cancer Inst. 2000;92(3):205-216.
Cancer Management and Research

\section{Publish your work in this journal}

Cancer Management and Research is an international, peer-reviewed open access journal focusing on cancer research and the optimal use of preventative and integrated treatment interventions to achieve improved outcomes, enhanced survival and quality of life for the cancer patient. The manuscript management system is completely online and includes

\section{Dovepress}

a very quick and fair peer-review system, which is all easy to use. Visit http://www.dovepress.com/testimonials.php to read real quotes from published authors. 